\title{
EL LIBERALISMO DE CARLOS NINO, ENTRE EL PERFECCIONISMO Y EL COMUNITARISMO*
}

\author{
MaRTín OLIVEIRA \\ Universidad de Buenos Aires - CONICET - Grupo de Filosofía Política
}

\section{Resumen}

En este trabajo trato de demostrar que Nino no logra articular un liberalismo completamente antiperfeccionista en su respuesta al comunitarismo. Para ello, parto de reconstruir el diagnóstico que Nino ofrece de la crítica comunitarista al liberalismo y señalo que para darle respuesta se compromete con una concepción particular del bien basada en la idea de autorrealización autónoma. En la siguiente sección desarrollo la manera en la que esa concepción del bien da forma al liberalismo de Nino y, en el apartado siguiente, la confronto con un experimento mental destinado a evaluar si es capaz de bloquear distintas políticas estatales de corte perfeccionista. En la última sección ofrezco unas breves conclusiones respecto al modo en el que la adopción de una concepción del bien particular limita las pretensiones antiperfeccionistas de su teoría liberal.

PALABRAS CLAVE: Autonomía; Liberalismo comprehensivo; Perfeccionismo.

\begin{abstract}
In this paper I attempt to prove that Nino does not succeed in developing a fully antiperfectionist account of liberalism as an answer to the communitarian challenge. I begin with a reconstruction of Nino's diagnosis of the communitarian challenge to liberalism and point out that, in order to answer it he commits himself to a particular conception of the good based on the idea of autonomous self-realization. In the next section I develop the way by which that conception of the good shapes Nino's liberalism. I further challenge his liberalism with a mental experiment designed to asses if it is capable of blocking several perfectionist State policies. In the last section I offer some brief remarks on the way the adoption of a particular conception of the good limits the antiperfectionist scope of Nino's liberalism.
\end{abstract}

KEY WORDS: Autonomy; Comprehensive Liberalism; Perfectionism.

\section{Introducción}

En muchas ocasiones se ha resaltado la figura de Carlos Nino como la de un intelectual público cuyas posturas políticas y convicciones personales no soslayaban su rigor argumentativo y su lucidez conceptual.

* Agradezco el apoyo y los valiosos comentarios de Julio Montero, así como las sugerencias del evaluador anónimo de Análisis Filosófico. 
Creo que, en ese sentido, podemos afirmar dos cosas sobre Nino. En primer lugar, que se encontraba comprometido con una serie de valores y derechos liberales básicos, como pueden ser el de la libertad de expresión, la autonomía individual o la neutralidad estatal frente a los planes de vida de los ciudadanos. Y que, al mismo tiempo, no dejó de ofrecer argumentos tan creativos como originales para defenderlos, intentando siempre partir de premisas que todo interlocutor dispuesto al diálogo racional pudiera aceptar.

Por dichas razones considero que un homenaje apropiado al pensamiento de Nino debería ser capaz de ponderar el modo en el que ofrecía argumentos e intentaba contestar incluso a las posiciones que rechazaba de plano. El objetivo puntual, entonces, de este trabajo es mostrar cómo la teoría liberal de Nino tomó forma a partir de sus respuestas, por una parte, al comunitarismo y, por la otra, al perfeccionismo. Estas dos posiciones resultan representativas de las alternativas conceptuales entre las que se ubica el liberalismo, tal como queda plasmado en el paradójico diagnóstico que hace Ronald Dworkin de las acusaciones que se le imputan a la corriente liberal y al que Nino apela en diversas ocasiones: "Dworkin menciona dos acusaciones a la concepción liberal de la sociedad que son, aparentemente, incompatibles entre sí; una es que el liberalismo es escéptico respecto de concepciones de lo bueno; la otra es que es autocontradictorio porque el mismo incluye una concepción de lo bueno" (Nino 2007b, p. 36). En el contexto de la discusión con el comunitarismo y el perfeccionismo, podemos articular esas acusaciones de la siguiente manera. Por una parte, el comunitarismo atribuye al liberalismo posiciones valorativas escépticas ${ }^{1}$ que son motivacionalmente vacías y resultan en versiones empobrecidas de la personalidad moral. Por la otra, si el liberalismo asume alguna concepción de lo bueno, debería tomar una posición perfeccionista y sostener que el Estado tiene la potestad de regular las normas sociales de acuerdo a dicha concepción de lo bueno. En consecuencia, debería prohibir o desalentar toda conducta contraria a la concepción de lo bueno asumida ${ }^{2}$. Pretender

${ }^{1}$ Cabe señalar que aunque pueda haber concepciones del liberalismo basadas en distintas formas de escepticismo moral, el liberalismo de John Rawls no solo no asume tesis escépticas sino que las rechaza. En Political Liberalism, por ejemplo, este filósofo argumenta que sostener posiciones escépticas respecto a las concepciones políticas de justicia sería contraproducente respecto al consenso superpuesto que busca alcanzar. Cf. Rawls (2005, p. 150).

2 Como se verá más adelante, ciertas versiones de la crítica comunitarista al liberalismo también acusan al liberalismo de asumir una concepción de lo bueno y de contradecirse al defender, a su vez, la neutralidad estatal. 
que el Estado se mantenga neutral entre planes de vida es contradictorio con el compromiso liberal con una concepción del bien. En dicho contexto, el liberalismo de Nino asume la defensa de las dos posiciones siguientes. Por una parte, toma al individuo como unidad última de justificación normativa, de manera que el liberalismo debe asegurar un núcleo básico e irrenunciable de derechos individuales. Y, por la otra, se compromete con una posición antiperfeccionista que afirma la neutralidad del Estado y la no intervención de terceros frente a la elección de los planes de vida de las personas.

Trataré de demostrar que, sin embargo, Nino no logra articular un liberalismo completamente antiperfeccionista en su respuesta al comunitarismo. Para ello, parto de reconstruir el diagnóstico que Nino ofrece de la crítica comunitarista al liberalismo y señalo que para darle respuesta se compromete con una concepción particular del bien basada en la idea de autorrealización autónoma. En la siguiente sección desarrollo la manera en la que esa concepción del bien da forma al liberalismo de Nino y, en el apartado siguiente, la confronto a un experimento mental destinado a evaluar si es capaz de bloquear distintas políticas de corte perfeccionista. En la última sección ofrezco unas breves conclusiones respecto al modo en el que la adopción de una concepción del bien particular limita las pretensiones antiperfeccionistas de su teoría liberal.

\section{Nino frente al desafío comunitarista}

Aunque podría sugerirse que las principales tesis de la crítica comunitarista al liberalismo quedaron agotadas tras una serie de argumentos entrecruzados durante la década de los ochenta ${ }^{3}$, parece difícil negar que ellas contribuyeron a robustecer el constructo teórico que pretendían derrotar. Indudablemente, el comunitarismo ofreció razones lo suficientemente persuasivas como para que John Rawls modifique la postura que defendía en $A$ Theory of Justice y llegue a las tesis presentes en Political Liberalism. Podría decirse que Dworkin tuvo una reacción semejante. Su versión del liberalismo basada en una concepción de la igualdad, que postula por primera vez en Foundations of Liberal Equality, es una alternativa a la "estrategia de discontinuidad" rawlsiana que

\footnotetext{
${ }^{3}$ Recordemos que los principales textos asociados al comunitarismo datan de fines de los setenta y principios de los ochenta: Hegel and the Modern Society, de Charles Taylor fue publicado en 1979, After Virtue de Alasdair MacIntyre en 1981, Liberalism and the Limits of Justice de Michael Sandel en 1982 y finalmente Spheres of Justice de Michael Walzer fue publicado en 1983.
} 
configura la respuesta de este último filósofo al desafío comunitarista. No fue menos el caso de Nino, que si acaso no había desarrollado una teoría propia del liberalismo antes de la crítica de los filósofos comunitaristas, sin dudas las utilizó para dar forma a su propia versión del liberalismo.

Según Nino, la crítica comunitarista parte de identificar algunos rasgos básicos del liberalismo para luego poner en cuestión cada uno de ellos. A los efectos de observar cómo los argumentos comunitaristas perfilan la posición de Nino, vale la pena transcribir ambos pasajes. La caracterización comunitarista del liberalismo, en la reconstrucción del propio Nino, es la siguiente:

primero, [1] la idea de que la moral está compuesta fundamentalmente por reglas que serían aceptadas por cualquier individuo racional en circunstancias ideales; en segundo término, [2] el requisito de que esas reglas sean neutrales respecto a los intereses de los individuos; en tercer lugar, [3] la exigencia de que las pautas morales sean también neutrales en relación a las concepciones de lo bueno que los individuos pueden sustentar; en cuarto término, [4] la idea de que los agentes morales destinatarios de tales reglas son los individuos humanos y no, por ende, entes colectivos; y finalmente, [5] la exigencia de que las reglas morales sean aplicadas del mismo modo a todos los individuos humanos, cualquiera sea su contexto social (Nino 1989, p. 138. Se ha agregado la enumeración entre corchetes.).

Por otra parte, la crítica comunitarista ${ }^{4}$ sostiene que el liberalismo se compromete con (a) una concepción "empobrecida y ficticia" de la persona moral a los efectos de defender la independencia de lo correcto respecto a lo bueno, (b) una concepción "noumenal" de la persona moral desvinculada de sus fines para defender la tesis de la neutralidad, (c) un conjunto de derechos individuales que son insostenibles sin una concepción del bien, y solapadamente con (d) una concepción del bien basada en la satisfacción de preferencias subjetivas que es problemática en sí misma e inconsistente con la tesis de la neutralidad (Nino 1989, p. 139). Como resultado de la caracterización comunitarista del liberalismo

4 Resulta interesante comparar la reseña que elabora Nino de la crítica comunitarista, con los cinco puntos de "parecido familiar" que Mulhall y Swift encuentran en ella (Mulhall y Swift 2004, pp. 158-159). Las distingue el hecho de que Nino no menciona la crítica comunitarista contra el pretendido universalismo de la teoría liberal. Este, sin embargo, parece claramente expuesto en los puntos primero y quinto de la caracterización del liberalismo por parte del comunitarismo en la cita previa. 
y sus críticas, Nino sugiere que el comunitarismo se resume en los siguientes cuatro apartados:

En primer lugar, la derivación de los principios de justicia y corrección moral de una cierta concepción de lo bueno. En segundo término, una concepción de lo bueno en que el elemento social es central e incluso prevalente. En tercer término, una relativización de los derechos y obligaciones de los individuos a las particularidades de sus relaciones con otros individuos, a su posición en la sociedad y a las peculiaridades de esta ${ }^{5}$. Finalmente, una dependencia de la crítica moral respecto a la práctica moral de cada sociedad, tal como aquella se manifiesta en las tradiciones, convenciones e instituciones sociales (Nino 1989, p. 141).

Dadas las críticas comunitaristas, Nino hace dos concesiones que, a su juicio, no menoscaban las principales tesis de la posición liberal ${ }^{6}$. La primera consiste en asumir la cuarta tesis del comunitarismo, esto es, la dependencia de la crítica moral respecto a la práctica moral. Sabemos, sin embargo, que la práctica que Nino resalta es la del discurso moral como herramienta para la adopción libre de principios intersubjetivos, la solución de conflictos y el acercamiento de posiciones diversas. Lo que parecería un recurso al relativismo solo lo es en manera muy moderada puesto que en la práctica del discurso moral, tal como la caracteriza Nino, los juicios morales dependen de las convenciones propias de la misma práctica ${ }^{7}$. Estas pueden resumirse en "un cierto sistema de conceptos

${ }^{5}$ Uno podría objetar la estrecha asociación que Nino hace entre el comunitarismo y el relativismo moral. Por mencionar un ejemplo, Martha Nussbaum ha intentado reconstruir la teoría aristotélica de las virtudes, de la que también hace uso MacIntyre, de modo que el desarrollo de ellas no sea relativo a criterios locales o internos de cada comunidad. Cf. Nussbaum (1988).

${ }^{6}$ Sí lo hacen, de acuerdo a Nino, las concesiones de Rawls al comunitarismo. Concluyendo su evaluación de las modificaciones que el autor de A Theory of Justice introdujo a su teoría, comenta: "este relativismo no permite dar razones para adoptar una cultura democrática, y ni siquiera permite resolver los conflictos que se presentan en el marco de esa cultura fuera de los límites del consenso que se da de facto; lejos de tener una misión práctica la filosofía política tendría la función meramente contemplativa de certificar las coincidencias y disidencias que de hecho se dan en el ámbito social" (Nino 1989, pp. 142-143). Ahora bien, un examen minucioso de la práctica del discurso moral tal como la caracteriza Nino podría sugerir que ambas respuestas al comunitarismo son mucho más semejantes de lo que él supone.

${ }^{7} \mathrm{Me}$ ha sido señalado que esta concesión de Nino parece comprometerlo con una forma ingenua de universalismo moral antes que con un acercamiento a las posiciones comunitaristas. Si bien parece incuestionable que las características estructurales que 
-como los de justo, razonable, etc.-, determinadas implicaciones conversacionales y ciertos presupuestos valorativos que están conectados a las funciones inherentes a la práctica misma de la discusión moral, además, obviamente, de que están involucrados en todo discurso o razonamiento" (Nino 1989, p. 145). En un giro distintivamente liberal, una consecuencia de esta caracterización es que la práctica crítica tiene un alcance casi ilimitado de no ser por la imposibilidad de ejercer una crítica de la práctica crítica sin circularidad ${ }^{8}$.

Ahora bien, la práctica del discurso moral no resulta suficiente para responder al comunitarismo. En un eco de las críticas que Hegel realizó al imperativo categórico kantiano, Nino advierte que si se compromete con una delimitación meramente formal de esa práctica (como lo son la universalidad o la imparcialidad como condición de la aceptabilidad de principios), ella no sería capaz de derivar ningún principio moral substantivo. Esto lo lleva a realizar la segunda concesión al comunitarismo, que consiste en afirmar que el liberalismo, al menos en la versión que él defiende, se encuentra comprometido con una concepción del bien particular. En primera instancia dicha concepción del bien consiste en el valor de la autonomía ${ }^{9}$, entendida como libre elección de principios y planes de vida. Una de las consecuencias normativas que tiene la adscripción del valor de la autonomía a la práctica del discurso moral es una concepción de la autonomía moral autolimitada. En efecto, dado que el discurso moral está orientado a la obtención de principios morales de alcance intersubjetivo, el valor de la autonomía veda la adopción de cualquier principio que "restrinja la capacidad de elección de otros". Por otra parte, cuando no existe la posibilidad de que la adopción

Nino destaca del discurso moral configuran una forma de universalismo moral, él parece insistir en que el discurso moral es una práctica de la que todos, incluso los críticos comunitaristas, somos participantes. Así, la crítica moral se acerca a la práctica moral, puesto que para Nino esta última es una práctica con características universalistas.

${ }^{8}$ En este sentido, Nino parece convencido de que no existe una práctica o lenguaje más profundos desde el que se pueda ejercer una crítica, sin circularidad, de la práctica crítica tal como él la articula. Cf. Nino (1989, p. 145).

${ }^{9}$ Es preciso mencionar que existe un abundante corpus bibliográfico sobre el concepto de autonomía y su vinculación con la filosofía política. Por mencionar algunos ejemplos sobresalientes, Gerald Dworkin ha escrito un importante libro sobre la autonomía y su relación con la coerción justificada. A su vez, Joseph Raz ha hecho de la autonomía un punto central de su liberalismo perfeccionista. John Christman y Joel Anderson, finalmente, han editado un volumen con distintos ensayos muy esclarecedores sobre el concepto. Cf. Dworkin (1997), Raz (1988) y Christman y Anderson (2005). En lo que sigue del trabajo no revisaré distintas presentaciones del concepto, sino que me limitaré a reconstruir el que utiliza Nino, esto es, el de autorrealización autónoma. 
de un principio moral o un ideal personal restrinja la capacidad de elección de otras personas, el valor de la autonomía se configura como autonomía personal (Nino 1989, p. 148).

Sin embargo, Nino advierte que apelar solamente al valor de la autonomía tampoco es satisfactorio, dado que esta no provee razones para actuar. Si el liberalismo requiere alguna concepción del bien que sea capaz de derivar de ella un núcleo de derechos típicamente liberales pero que a su vez no sea motivacionalmente vacía, debe adscribir el valor de la autonomía a algún otro valor que pueda operar como razón para actuar. Es entonces cuando Nino apela a la idea de autorrealización ${ }^{10}$, que caracteriza de la siguiente manera:

Realizarse implica desarrollar las capacidades con que empíricamente cuentan los individuos [...] Nosotros valoramos positivamente a quienes desarrollan algunas de estas capacidades en los grados máximos posibles sin precluir totalmente el desarrollo de las otras [...] Las posibilidades de combinar el desarrollo de las diferentes capacidades son infinitas y valoramos la creatividad en la elección de alternativas. (Nino 1989, pp. 149-150).

Según entiendo, Nino afirma que la autorrealización es una concepción del bien valiosa y operativa cuando satisface las tres siguientes condiciones. Por una parte, (i) cuando hace posible el desarrollo de una o varias capacidades, (ii) cuando el llevar a cabo (i) no pone en riesgo las otras capacidades al punto de anularlas y finalmente (iii) cuando la elección de alternativas en cuanto a las capacidades a desarrollar es autónoma, o sea, propia de cada individuo y ajena a la intervención de terceros. Mientras que los elementos (i) y (ii) caracterizan la autorrealización, el punto (iii) lo vincula a la autonomía sosteniendo que la "creatividad en la elección de alternativas" hace valiosa a la autorrealización ${ }^{11}$ (Nino 1989, p. 150).

${ }^{10}$ Del mismo modo en el que establecí algunos reparos respecto al tratamiento de la autonomía en la nota anterior, corresponde hacer lo propio con el concepto de autorrealización como desarrollo de capacidades que utiliza Nino. En particular, el autor notó que todavía quedaba pendiente profundizar tanto el tratamiento del concepto como su vínculo con el razonamiento moral. Cf. Nino (1989, p. 151).

Aunque podrían surgir dudas respecto a la relevancia de "capacidades empíricas" para el razonamiento moral o, por caso, el desarrollo perverso de las mismas, en este trabajo me limito a analizar la letra del planteo de Nino y no sus posibles extensiones o ajustes.

${ }^{11}$ En lo que sigue de este artículo adopto una versión débil de la idea del desarrollo de las capacidades, esto es, una que no supone como condición necesaria del valor de 


\section{La concepción liberal de Nino}

Si mi reconstrucción de la posición de Nino es correcta, su defensa del liberalismo adopta la forma de un liberalismo antiperfeccionista comprehensivo basado en una concepción muy particular de la autorrealización. Ahora bien, para determinar su alcance, resulta conveniente puntualizar cómo los diversos elementos reseñados en la sección anterior articulan esta variante de la teoría liberal. Jonathan Quong propuso recientemente el siguiente par de preguntas como criterio de distinción de las teorías liberales: (1) ¿ La filosofía política liberal debe estar basada en algún idea particular de lo que constituye una vida humana valiosa o alguna otra creencia metafísica? y (2) ¿Es permisible a un Estado liberal promover o desincentivar algunas actividades, ideales o formas de vida por razones relativas a su valor inherente o intrínseco o por razones relativas a alguna otra creencia metafísica? (Quong 2011, p. 15). Una respuesta positiva a la primera pregunta hace comprehensiva a cualquier variante del liberalismo, mientras que una respuesta negativa la hace político. Una respuesta positiva por parte del liberalismo a la segunda pregunta lo hace perfeccionista, mientras que una respuesta negativa lo hace antiperfeccionista.

Creo que la segunda concesión que Nino hace al comunitarismo sirve de respuesta la primera pregunta de Quong. En efecto, de acuerdo a lo que se ha señalado antes, Nino afirma que el liberalismo debe apelar a una concepción del bien basada en la idea de autorrealización autónoma para poder defender derechos liberales y la democracia a través del discurso moral. Como señala Gerald Gaus, este elemento comprehensivo no es en absoluto nuevo, sino que ha sido frecuentemente la piedra de toque del liberalismo como teoría de la buena vida, identificable incluso en la tercera parte de A Theory of Justice de Rawls o también en el liberalismo clásico de John Stuart Mill. Más aún, la idea del desarrollo "armónico" de las capacidades ya había sido presentada por el filósofo inglés en su comparación de la naturaleza humana con "un árbol, que

la autorrealización que la capacidad sea maximizada. Considero que adoptar una versión fuerte de ese requisito en todo caso refuerza el argumento que se ofrece más adelante, pero que no es estrictamente necesario para su presentación.

Debemos notar que el requisito (ii) guarda una estrecha semejanza a ciertas concepciones del bien basadas en el desarrollo armónico de ciertas virtudes. Aunque se hable de capacidades y mientras estas no sean puramente políticas, al estilo del sentido de justicia y la capacidad para desarrollar una concepción del bien de Rawls, ya se advierte una veta perfeccionista en el planteo de Nino. 
requiere crecer y desarrollarse en todas direcciones, de acuerdo a la tendencia de las fuerzas interiores que lo hacen un ser viviente" (Mill 1962, p. 188 y Gaus 2004, p. 102). Lo distintivo de la propuesta de Nino consiste en hacer de la autonomía una condición necesaria para que la autorrealización de un individuo resulte valiosa.

La concepción de lo bueno basada en la autorrealización en conjunto con el requisito de autonomía son los dos elementos conceptuales que le permiten a Nino enfrentar a la vez las acusaciones a la teoría liberal que señalaba Dworkin como la segunda pregunta de Quong y obtener conclusiones antiperfeccionistas ${ }^{12}$. En efecto, frente al dilema que, por un lado, acusaba al liberalismo de sostener posiciones valorativas escépticas $y$, por el otro, de ser autocontradictorio porque defiende a la vez una concepción del bien y la neutralidad estatal, el liberalismo de Nino apela a la distinción entre concepciones de lo bueno y planes de vida derivados de ellas. Por una parte admite comprometerse con una concepción de lo bueno -la autorrealización autónoma- y refuta el cargo de escepticismo valorativo y, por la otra, justifica de manera no contradictoria la neutralidad del Estado frente a los diversos planes de vida apelando al valor de la libre elección en el desarrollo de las capacidades -la autonomía- (Nino 2007a, p. 209). La respuesta al segundo cuerno del dilema es también una respuesta negativa a la segunda pregunta de Quong, en tanto un Estado liberal que afirma el valor de la autonomía personal no debe ser perfeccionista.

\section{El problema de la autorrealización}

Ahora bien, aunque Nino advirtió que la vinculación entre los valores de la autonomía y el de la autorrealización resultaba "un tema enormemente complejo" y que solo recibió un tratamiento tentativo (Nino 1989, p. 149), creo que es posible dudar de que una exploración más exhaustiva de esa conexión conceptual hubiera sido fructífera. En lo que resta de este trabajo intentaré mostrar que el valor de la autorrealización

12 Aquí debemos señalar nuevamente que el Political Liberalism de Rawls es el ejemplo paradigmático de una posición liberal antiperfeccionista política. Para examinarla en detalle resultan útiles, además del libro de Quong antes citado, por una parte, el trabajo de Hugo Seleme Neutralidad y justicia; por la otra, Legitimidad política y neutralidad estatal de Mariano Garreta Leclercq. En el primero, el autor desarrolla los cambios en la teoría rawlsiana desde A Theory of Justice hacia Political Liberalism, en particular a partir de las críticas de Sandel. Mientras que, en el segundo, se estudian diversos argumentos a favor de la neutralidad estatal, entre ellos y con especial detenimiento, los rawlsianos. Cf. Seleme (2004) y Garreta Leclercq (2007). 
no necesariamente colabora en la defensa de las posiciones que Nino adjudica al liberalismo sino que exige modificar, al menos, una de ellas.

De acuerdo a la teoría de Nino, el discurso moral es la plataforma de justificación de las normas intersubjetivas. Ahora bien, todo participante leal en el discurso moral debe asumir como prerrequisito normativo el valor de la autonomía moral. Sin embargo, dicho valor por sí solo, en conjunción con condiciones formales y performativas del discurso, no permite la derivación de principios morales substantivos. En efecto, para poder incorporar razones últimas para la acción en el discurso moral todo participante debe asumir el valor de la autorrealización calificado por la autonomía.

Según se estableció previamente, solo es valiosa la autorrealización si satisface las tres condiciones siguientes: (i) desarrolla una o varias capacidades, (ii) el llevar a cabo (i) no pone en riesgo las otras capacidades al punto de anularlas, y finalmente (iii) la elección de alternativas en cuanto a las capacidades a desarrollar es autónoma, o sea, propia de cada individuo y ajena a la intervención de terceros. Las primeras dos condiciones (i) y (ii) establecen los requisitos de la autorrealización mientras que (iii) es la cláusula antiperfeccionista basada en la autonomía.

Llegado este punto, debemos interrogarnos qué es lo que impide, dada la reconstrucción de la práctica del discurso moral ofrecida arriba, que se deriven de ella normas intersubjetivas de corte perfeccionista. Procederé por medio de un experimento mental en el que se contrastan distintas políticas perfeccionistas con los presupuestos normativos del discurso moral. Si alguno de los presupuestos normativos entra en conflicto con la política propuesta, entonces esta última no es admisible como norma estatal. En cambio, si una política perfeccionista no viola ninguno de los presupuestos normativos del discurso moral, sería admisible como norma estatal. Debemos observar que si de la práctica del discurso moral como la he reconstruido no es posible derivar ninguna política perfeccionista, entonces el liberalismo de Nino sería antiperfeccionista. Por otra parte, si de ella se derivara al menos una política perfeccionista, el liberalismo de Nino no sería completamente antiperfeccionista.

Las siguientes podrían ser distintas políticas perfeccionistas propuestas como normas estatales: (a) El Estado debe forzar a todos los ciudadanos a elegir un plan de vida "p" que desarrolla armónicamente sus capacidades en un grado $n+1$ antes que un plan de vida "q" que solo lo hace en un grado n; (b) El Estado debe prohibir la elección un plan de vida "r", dado que es el que menos desarrolla armónicamente las capacidades; (c) El Estado debe incentivar, por medio de subsidios, la elección de planes 
de vida que desarrollan armónicamente alguna capacidad sin anular a las demás, pero que sin este incentivo no serían elegidos; (d) El Estado debe prohibir la adopción de un plan de vida "s" que por mor del desarrollo de una capacidad, anula otras; (e) El Estado debe forzar a los ciudadanos a elegir algún plan de vida que desarrolle al menos una capacidad en algún grado y prohibir todo plan de vida que no lo haga, aunque tampoco anule otras.

La política perfeccionista (a) satisface las cláusulas (i) y (ii) pero el incentivo a la elección de un plan de vida sobre otro parece violar (iii) en tanto que la elección del plan de vida "p" por parte de los individuos se habría visto influenciada por la acción de terceros y, en consecuencia, la autorrealización consecuente no sería valiosa. Respecto a la política perfeccionista (b) cabe observar que un plan de vida "r" que no desarrolle superlativamente ninguna capacidad pero tampoco las anule no viola ni la cláusula (i) ni la (ii), pero sin duda su prohibición afecta la elección autónoma de los individuos y viola la cláusula (iii).

La política (c) representa un caso especial de norma perfeccionista, en tanto que, por una parte pretende influir en los ciudadanos de manera no coercitiva. Por la otra, es el ejemplo de una política estatal que toda teoría antiperfeccionista comprehensiva necesita bloquear, dado que de otra manera incorporará de manera indirecta elementos perfeccionistas (Hurka 1995, pp. 48-50; Garreta Leclercq 2007, pp. 59-65 y Quong 2011, p. 25). Un argumento frecuente en defensa de la posición antiperfeccionista comprehensiva consiste en señalar que el incentivo nocoercitivo de ciertos planes de vida, por ejemplo a través de subsidios, requiere del cobro de impuestos. Esta última es una medida que sin duda tiene un carácter coercitivo. Sin embargo, afirmar que el subsidio de planes de vida por medio de impuestos es una política coercitiva que no puede estar justificada en un Estado neutral, configura una pendiente resbaladiza en la que la justificación del cobro de impuestos con fines redistributivos se vuelve enormemente compleja (Hurka 1995, p. 50). Creo que, a pesar de la objeción, la posición de Nino es capaz de defender el cobro de impuestos con fines redistributivos sin comprometerse con la justificación de este tipo de políticas perfeccionistas. Si bien no es posible desarrollar por completo el argumento igualitarista de Nino basado en el principio de inviolabilidad de la persona (Nino 2007b, pp. 52-67), cabe señalar lo siguiente. En el artículo "Autonomía y necesidades básicas", Nino defiende la idea de que aquello que el Estado debe distribuir de manera igualitaria no es el grado de satisfacción de cualquier plan de vida elegido a partir de un ejercicio abstracto de imaginación, sino ciertas necesidades básicas entendidas como las capacidades que hacen posible 
la elección autónoma de planes de vida materializables (Nino 2007b, pp. 108-109). En definitiva, lo que el Estado se encuentra justificado en maximizar es la autonomía de cada individuo hasta el grado en el que el aumento de la autonomía de un individuo disminuya la de otro. Pero en tanto se entiende que la autonomía configura el conjunto de las necesidades básicas para elegir un plan de vida materializable, esto no implica el incentivo a la elección de ciertos planes de vida particulares.

Considero, sin embargo, que el discurso moral se encuentra frente a graves inconvenientes a la hora de bloquear políticas perfeccionistas como la (d) o la (e). En particular, podemos observar que un plan de vida "s" que implica el desarrollo de una o varias capacidades al costo de anular otras, aun elegido de manera autónoma, no es valioso porque viola la cláusula (ii). Asimismo, un plan de vida que no desarrolle al menos en un mínimo grado una capacidad, aun siendo elegido de manera autónoma, tampoco es valioso porque no satisface la cláusula (i) de las condiciones de autorrealización. Las políticas perfeccionistas (d) y (e) apuntan precisamente a asegurar que solo sean elegidos planes de vida que conduzcan a la autorrealización y, por ello, sean valiosos. No imponen a los ciudadanos un determinado plan de vida valioso, sino que los obligan a elegir algún plan de vida valioso. Ahora bien, el principal recurso del discurso moral para bloquear medidas perfeccionistas consistía en la cláusula (iii) que requería la elección autónoma de algún plan de vida valioso. Sin embargo, prohibir la adopción de planes de vida que no conducen a la autorrealización y, en consecuencia, son carentes de valor y obligar a los ciudadanos a elegir algún plan de vida valioso, no viola (iii), esto es, la elección autónoma de algún plan de vida valioso.

Podría pensarse que si renunciamos a incluir entre los presupuestos normativos del discurso moral una concepción del bien basada en la autorrealización, políticas perfeccionistas como la (d) o la (e) serían bloqueadas y se preservaría el componente liberal de la autonomía. Sin embargo, considero que no es posible intentar dicha solución. En efecto, como señalé más arriba, la práctica del discurso moral se encuentra comprometida y asume como presupuesto normativo el ideal de la autorrealización. Dado que la autonomía no provee razones últimas para actuar, Nino la ha incorporado como una condición esencial de la autorrealización. Si uno renuncia al ideal de la autorrealización, habría que ofrecer una justificación independiente del ideal de autonomía y, a su vez, explicar, a partir de este u otro ideal, cómo provee razones últimas para actuar. En el caso de que mi reconstrucción de la concepción del bien con la que se compromete Nino -la autorrealización autónoma- y su interacción con el discurso moral sean correctas, cabría concluir que su 
liberalismo es menos antiperfeccionista de lo que pretende. En particular, la práctica del discurso moral es incapaz de bloquear al menos dos medidas de corte perfeccionista que podría llegar a adoptar el Estado y que involucran su intervención sobre terceros.

\section{Conclusión}

Es plausible pensar que las tesis comunitaristas están lejos de tener las consecuencias nacionalistas y totalitarias que Nino creía ver en ellas (Nino 1989, pp. 141-142). Si el comunitarismo no es una corriente que agotó su potencia conceptual a fines de los ochenta, como se mencionó previamente, tampoco se puede observar que haya producido teorías positivas hoy en boga. Antes bien, creo que es conveniente coincidir con Michael Walzer y sostener que el comunitarismo depende conceptualmente del liberalismo y que su mayor virtud consiste en la crítica a y las "correcciones" que produce en esta última corriente (Walzer 1990, p. 21).

En ese sentido, podemos considerar como un ejemplo de ello a la teoría del propio Nino. Dado su propósito de asegurar un núcleo de derechos liberales básicos frente a posturas relativistas, intentó ofrecer una justificación del liberalismo mordiendo la bala de una de las críticas comunitaristas y asumiendo, entonces, una concepción del bien. Con el recurso al valor de la autonomía, la teoría liberal de Nino resguarda, en gran medida, la neutralidad del Estado y los derechos que quería proteger. Pero dado que en el contexto del razonamiento práctico la autonomía no es suficiente, es necesario asociarla a una concepción del bien mucho más densa. Esa concepción del bien, sin embargo, supone poner un límite al valor de la autonomía y limitar el alcance antiperfeccionista de la teoría liberal de Nino. No resulta paradójico entonces sugerir que su estrategia argumentativa exhibe una estructura muy semejante a la del "razonamiento silogístico" con el que Charles Taylor intentaba mostrar los problemas del liberalismo. Esto es, la idea de que para justificar derechos es necesario reconocer ciertas capacidades como valiosas y que, en consecuencia, hay que preservar y expandir dichas capacidades desde el Estado (Taylor 1985, p. 195 y Nino 1989, pp. 139-140).

\section{Bibliografía}

Christman, J. y Anderson, J. (2005), Autonomy and the Challenges to Liberalism, Cambridge, Cambridge University Press.

Dworkin, G. (1997), The Theory and Practice of Autonomy, Cambridge, 
Cambridge University Press.

Garreta Leclercq, M. (2007), Legitimidad política y neutralidad estatal, Buenos Aires, Eudeba.

Gaus, G. (2004), "The Diversity of Comprehensive Liberalisms" en Gaus, G. y Kukathas, C. (eds.), Handbook of Political Theory, Londres, Sage.

Hurka, T. (1995), "Indirect Perfectionism: Kymlicka on Liberal Neutrality", The Journal of Political Philosophy, 3(1), pp. 36-57.

Mill, J. S. (1962), Utilitarianism, On Liberty, Essay On Bentham, Westford, Meridian.

Mulhall, S. y Swift, A. (2004), Liberals and Communitarians, Oxford, Blackwell.

Nussbaum, M. (1988), "Non-Relative Virtues: An Aristotelian Approach", Midwest Studies in Philosophy, 13(1), pp. 32-53.

Nino, C. S. (1989), El constructivismo ético, Madrid, Centro de Estudios Constitucionales.

(2007a), Ética y derechos humanos, Buenos Aires, Astrea. (2007b), Derecho, moral y política II, Barcelona, Gedisa.

Quong, J. (2011), Liberalism without Perfection, Nueva York, Oxford University Press.

Rawls, J. (2005), Political Liberalism, Nueva York, Columbia University Press.

Raz, J. (1988), The Morality of Freedom, Oxford, Oxford University Press. Seleme, H. (2004), Neutralidad y justicia, Madrid, Marcial Pons.

Taylor, C. (1985), Philosophy and the Human Sciences, Philosophical Papers Vol. 2, Cambridge, Cambridge University Press.

Walzer, M. (1990), "The Communitarian Critique of Liberalism", Political Theory, 18(1), pp. 6-23.

Recibido el 14 de enero de 2015; aceptado el 12 de marzo de 2015. 